\title{
In-Flight Thrust Measurement using On-Board Force Sensor
}

\author{
Murat Bronz, ${ }^{*}$ Hector Garcia de Marina, ${ }^{\dagger}$ and Gautier Hattenberger ${ }^{\ddagger}$ \\ ENAC, F-31055 Toulouse, France \\ University of Toulouse, F-31400 Toulouse, France
}

\begin{abstract}
Preliminary results of an in-flight thrust measurement method are presented in order to determine the drag force. Flight experiments are conducted by an off-the-shelf aircraft which has been equipped with an autopilot system and additional sensors measuring the electric motor rotation speed, battery voltage, drawn current, and direct thrust of the motor. Several level cruise flights have been performed in order to measure the thrust, thus the drag force generated by the aircraft. The propulsion system used on the aircraft is first characterized in the wind-tunnel. The direct thrust measurement system which is on-board the aircraft presents a lot of noise coming from vibrations, characteristics of the sensor itself, and also the fuselage wake and propeller interaction. Hence a better estimation of the in-flight thrust and the resultant drag is obtained by sensor fusion of Gaussian signals.
\end{abstract}

\section{Introduction}

The use of small Unmanned Air Vehicles (UAVs) are increasing on daily basis. The possible applications are countless and not only limited with military but there are also civil applications such as surveillance, atmospheric research, ${ }^{1}$ search and rescue,${ }^{2,3}$ inspection, agriculture, etc... The compact size and ease of operation makes the small UAVs more attractive, however their relatively lower performance compared to bigger UAVs highly limits the endurance, range, and payload capabilities.

Therefore, enhancing the performances is a very active research topic and in that the aerodynamic characterization is one of the main issues. Optimization of the airframe specifically for a mission profile can result in big gains in flight performance and quality of the UAV as presented by Bronz and Hattenberger. ${ }^{4}$

Several other work investigated the identification techniques for small or larger UAVs. ${ }^{5-7}$ In most cases, the goal is to produce a state space model directly usable for applying advanced control theory, hence focusing on the dynamics. The work from Edwards ${ }^{8}$ proposes a simple and practical method to extract lift and drag coefficients from flight tests based on analysis on gliding phases. However, this last method is less applicable to smaller vehicles because of their small inertia, which makes them more vulnerable to external perturbations. Therefore the drag measurement remains hard to obtain.

\section{A. Present Work}

This study, being mainly a continuation of, ${ }^{9}$ focuses on obtaining in-flight thrust measurements through an on-board mechanism that uses a contact pressure sensor. The direct force sensor measurement during flight is fused with additional sources that estimates the force generated by the propulsion system. Airspeed of the vehicle is estimated with an extended Kalman filter. The propulsion system used on the aircraft is first characterized in the wind-tunnel as a function of propeller rotation speed (RPM), and the incoming freestream velocity. Then during the flight, the instantaneous measured RPM and the estimated airspeed are used to obtain the propeller thrust force. A second linear Kalman filter estimates the acceleration of the aircraft by using the velocity difference between the previous and the actual states, and estimates the resultant net force on the aircraft. Later on, this acceleration is used to correct the direct force measurement mechanism's values. The corrected measurement distribution is fused with the estimated thrust measurement

\footnotetext{
*Assistant Professor on Applied Aerodynamics, ENAC UAV Lab, Toulouse, France

${ }^{\dagger}$ Post-Doctoral Researcher on Systems and Control, ENAC UAV Lab, Toulouse, France

$\ddagger$ Assistant Professor on Flight Dynamics, ENAC UAV Lab, Toulouse, France
} 
distribution coming from the characterized propeller equation. Finally the difference between the fused measurement distribution and the estimated net force gives the total drag force distribution as a result.

The proposed methodology is designed mostly for aerodynamic drag force characterization of small UAVs, however it can also be used to shed light on the propeller and fuselage wake interaction mechanism, propellerwing and propeller-propeller interaction on complex systems such as distributed electric propulsion. ${ }^{10}$ The precision of the selected force sensor becomes a keypoint for the latter investigations.

The aircraft specifications and all of the on-board sensors are presented in Section II. Propulsion system characterization and the resultant coefficients are presented in the Section III. The proposed methodology for the in-flight thrust and drag force estimation is explained in the following Section IV. Preliminary flight test results and the feasibility of the in-flight thrust and drag force measurements are discussed in the Section V, including the calibration problems and all other sources of errors. The paper is finalized with final conclusion and perspectives for further improvements of the system.

\section{Instrumentation}

The aircraft used during the flight tests is an off-the-shelf frame commercially available for recreational use. General specifications of the aircraft are given in Table 1. It is made up of Elapor foam material, which makes it light weight and easy to repair, however it also limits the maximum take-off mass and the flight velocity due to limited strength. Take-off mass and the flight velocity are given mainly according to the mentioned reason on aircraft's structural properties.

\section{A. Autopilot and Sensors}

The Apogee a autopilot board running the Paparazzi Autopilot System, ${ }^{11,12}$ is used throughout the whole experimental flights. The Paparazzi system is a well known and proven open-source and open-hardware system, used by hundreds of individual user around the world.

The Apogee board has 3-axis gyroscope, accelerometer, magnetometer (MPU-9150), and a lowresolution barometer (MPL-3115A2). A GPS receiver module (Ublox-NEO-M8N) is added externally.

\begin{tabular}{lcl}
\hline Wing Span & 1.288 & {$[\mathrm{~m}]$} \\
Wing Surface Area & 0.27 & {$\left[\mathrm{~m}^{2}\right]$} \\
Mean Aero. Chord & 0.21 & {$[\mathrm{~m}]$} \\
Electric Motor & AXI $2212 / 26$ & \\
Prop Diameter & 0.228 & {$[\mathrm{~m}]$} \\
Take-off Mass & $0.7-2.0$ & {$[\mathrm{~kg}]$} \\
Flight Velocity & $10-25$ & {$[\mathrm{~m} / \mathrm{s}]$} \\
\hline
\end{tabular}

Table 1. General specifications of the Aircraft.

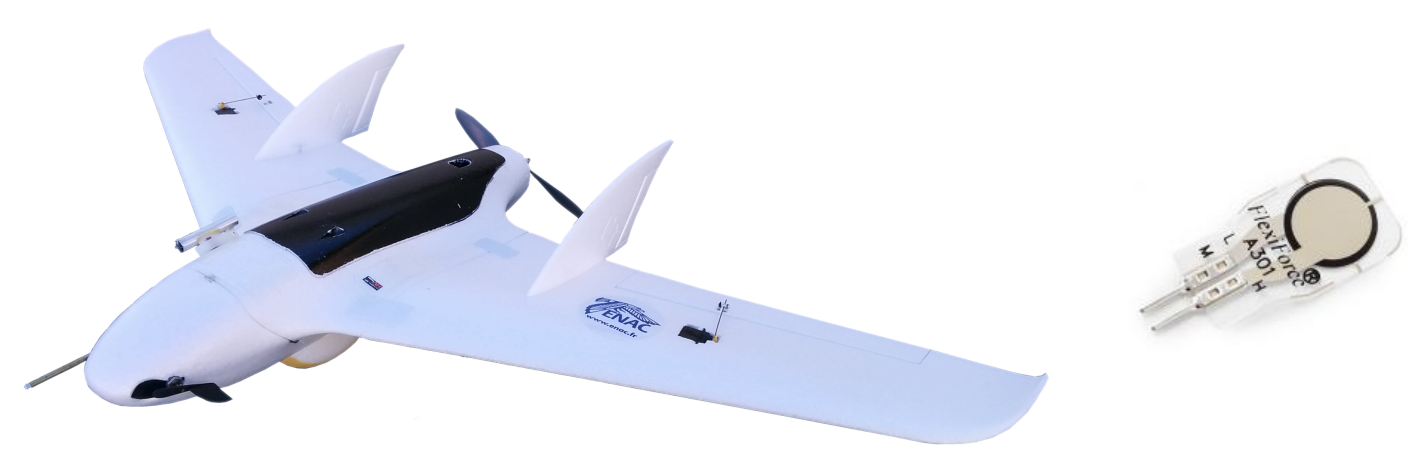

Figure 1. Mako aircraft general view on the left, and the FlexiForce A301 thin film force sensor on the right.

\section{B. On-board Force Sensor Mechanism}

A thin-film force sensor (FlexiForce A301) is integrated to the motor mount in order to measure the thrust force during the flight. In an ideal cruise condition without acceleration on any axis, the thrust force measurements will give directly the total drag force of the aircraft at that flight speed. The mounting of the sensor and the motor mount mechanism is shown in Figure 2.

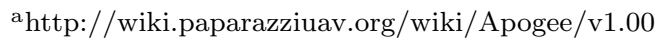




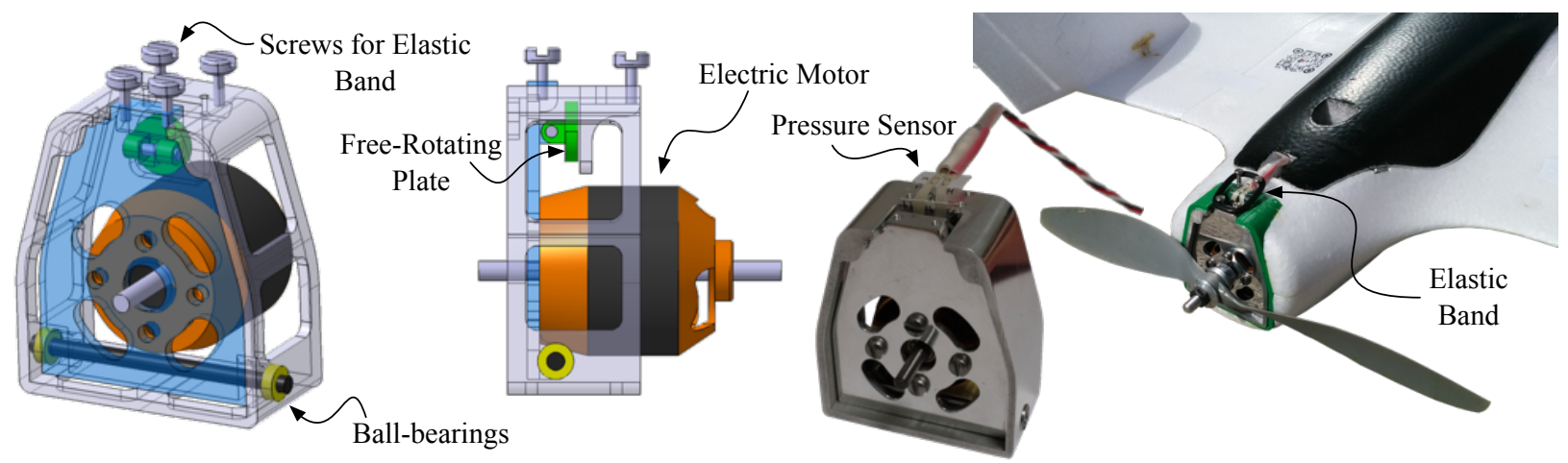

Figure 2. Developed mechanism for the on-board thrust force measurements is shown, starting from left to right with isometric and side CAD drawing views, CNC-manufactured real piece and its integration to the test aircraft.

\section{Propulsion System Characterization}

The propulsion system used on the aircraft is first mounted on a test-bench placed inside a wind-tunnel in order to obtain the performance characteristics. The thrust and some other quantities are measured for a set of different wind-tunnel speeds and varying propeller rotation speeds. For the current study, thrust measures are gathered and fitted into an equation as a function of wind-tunnel freestream velocity $V_{\infty}$ and propeller rotation speed as in revolution per minutes RPM. The resultant function serves as a thrust estimation function in order to be fused with in-flight thrust measurements. More detailed information on the measurements and characterization method can be found in. ${ }^{13}$

\section{A. Propulsion Test Bench}

Figure 3 shows the propulsion test bench. Two force sensors (SMD S- $100^{\mathrm{b}}$ ) are used to measure the propulsive force and the motor torque. ESC32v3 speed controller is used for controlling the rotation speed of the brushless motor and recording the voltage, current, and motor rotation speed data through its serial connection.
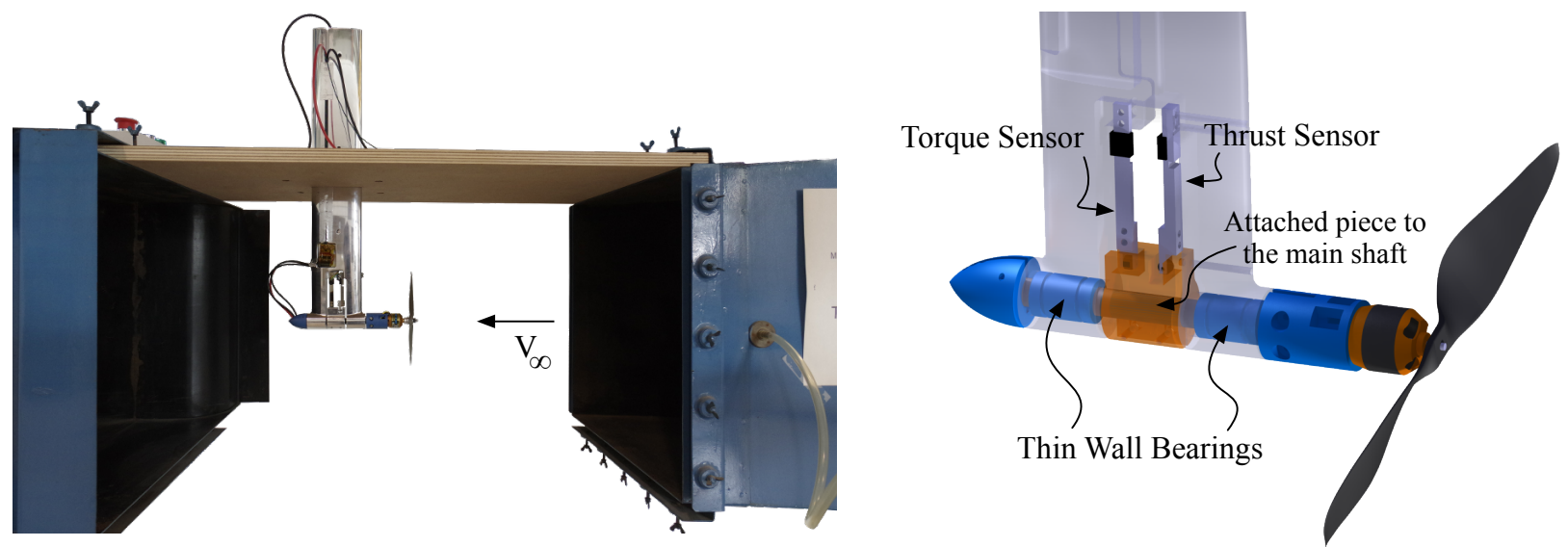

Figure 3. On the left, propulsion test bench mounted in the wind tunnel of ENAC, and on the right a detailed CAD drawing view of the sensor integration and the moving parts are shown.

In addition to the mechanical mounting of the motor and its propeller, an electronic board is required for the sensor signal conditioning. An in-house developed board is used for this, and then the analog signals are converted to 12 bit digital signal with an STM32-F4 micro controller and fed into the computer. A graphical interface is also developed in Python language, which allows to control the motor PWM command

\footnotetext{
$\mathrm{b}_{\text {www.smdsensors.com }}$
} 
and synchronize all the measurements and filters, making the process almost fully automated for the user (the wind tunnel speed is currently controlled by hand).

\section{B. Characterization}

The propulsion test bench have been placed in a wind tunnel and the measurements have been recorded at different airspeed from $0 \mathrm{~m} / \mathrm{s}$ (static thrust) up to $20 \mathrm{~m} / \mathrm{s}$. The resulting thrust versus RPM is shown in Figure 4. Maximum thrust at static condition is about $6 \mathrm{~N}$ and reduces down to $3 \mathrm{~N}$ at $20 \mathrm{~m} / \mathrm{s}$ flight speed.

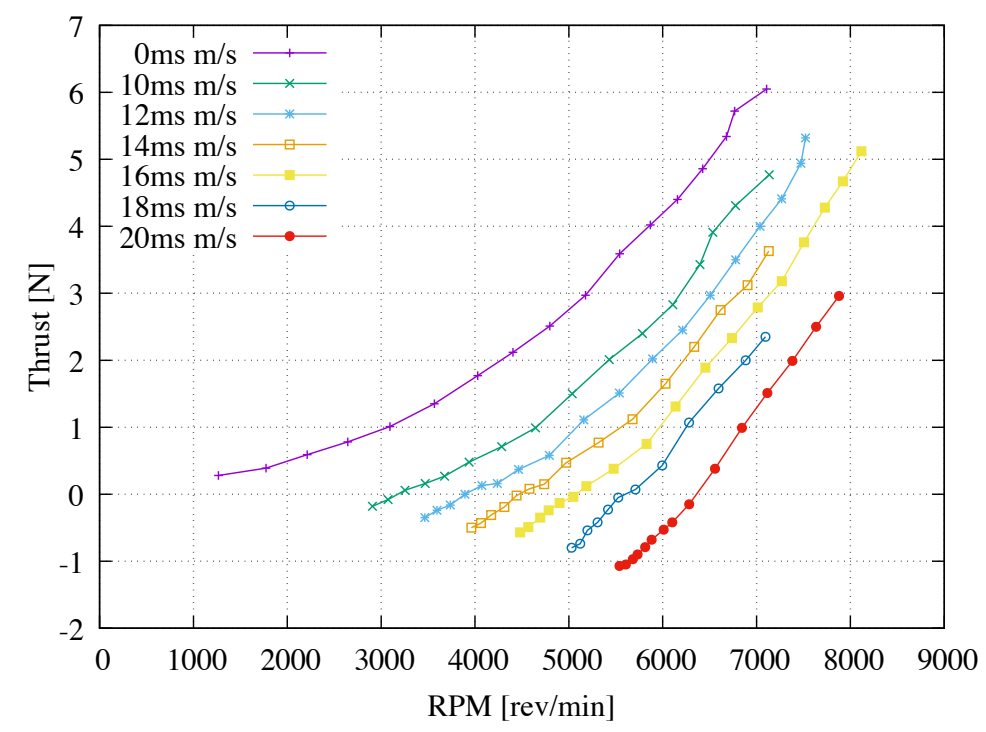

Figure 4. Thrust versus RPM at different airspeed from wind tunnel experiment are shown.

It can be seen that at higher speed, the motor is not generating positive thrust at low RPM values (at $20 \mathrm{~m} / \mathrm{s}$ it needs at least $70 \%$ of throttle) since the pitch of the propeller and the RPM at that throttle is not sufficient to have a positive local angle of attack on the blade section.

The $C_{T}$ coefficient can be extracted from these measurements using classical equation 1.

$$
T(J, \Omega)=T(\Omega, V)=\rho n^{2} D^{4} C_{T}\left(J, R_{e}\right)
$$

In addition, it is more desirable to present the thrust force as a function of flight speed and RPM, therefore $C_{T}(J, \Omega)$ can be written by using three coefficients $C_{T_{0}}, C_{T_{J}}, C_{T_{R P M}}$ as in Equation 2.

$$
C_{T}(J, \Omega)=\frac{T}{\rho n^{2} D^{4}}=C_{T_{0}}+C_{T_{J}} J+C_{T_{R P M}} R P M
$$

The resultant coefficients after a linear regression are shown in Table 2.

\begin{tabular}{ccccc}
\hline Prop & $C_{T 0}$ & $C_{T J}$ & $C_{T R P M}$ & $R^{2}$ \\
\hline APC 9x6 & $1.342 \mathrm{e}-01$ & $-1.975 \mathrm{e}-01$ & $7.048 \mathrm{e}-06$ & 0.983 \\
\hline
\end{tabular}

Table 2. Resultant coefficients for $C_{T}$ function.

\section{Methodology}

This section presents the signal processing algorithm for estimating the drag force of the aircraft. The signal flow is depicted in Figure 5. We use two Kalman filters that estimate the needed variables for calculating the set of forces that will be employed to obtain the drag force. 


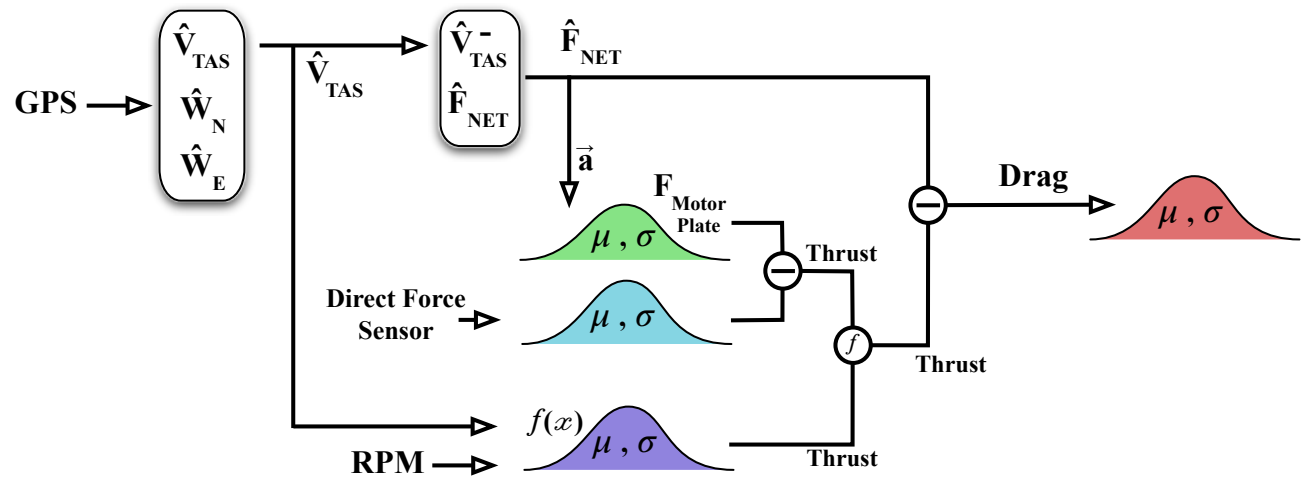

Figure 5. Main parts of the drag estimation method is presented.

\section{A. True airspeed and wind estimation}

The true airspeed is estimated from the ground speed and tracking angle measurements coming from the on-board GPS. The estimation is done via a discrete extended Kalman filter, as show by Edwards ${ }^{14}$ and Kahn, ${ }^{15}$ whose states are

$$
\hat{X}_{1}=\left[\begin{array}{lll}
\hat{V}_{T A S} & \hat{W}_{N} & \hat{W}_{E}
\end{array}\right]^{T},
$$

where $\hat{V}_{T A S}$ is the true airspeed and $\hat{W}_{N, E}$ are the North and East components of the estimated wind vector. The dynamics of $X_{1}$ are modelled as follows

$$
\hat{X}_{1}(k+1)=I \hat{X}_{1}(k),
$$

where $I$ is the identity matrix, i.e. we consider that between two time instants $k+1$ and $k$ the state vector $X_{1}$ remains constant, which is not a very conservative assumption since the GPS signal employed in the observation model is measured at $5 \mathrm{~Hz}$ frequency. However, in order to incorporate small variations of the state vector between two consecutive measurements, we consider the following process noise matrix $Q_{1}=1 \times 10^{-4} I$ for the Kalman filter.

The correction stage for the Kalman filter is based on the relationship between the inertial wind velocity vector $V_{w}$, airspeed inertial velocity vector $V_{a}$, and the inertial ground velocity vector $V_{g}$, namely

$$
V_{g}=V_{a}+V_{w},
$$

which gives us the following set of equations

$$
\begin{gathered}
N_{a}=V_{g_{n}}-\hat{W}_{N} \\
E_{a}=V_{g_{e}}-\hat{W}_{E} \\
\epsilon=\sqrt{N_{a}^{2}+E_{a}^{2}}-\hat{V}_{T A S},
\end{gathered}
$$

where $V_{g_{e}}$ and $V_{g_{n}}$ are the inertial ground speed vectors for north and east components that are available from the on-board GPS and $\epsilon$ is what we choose to be the observable in order to correct $\hat{X}_{1}$. Therefore the correction stage of the Kalman filter can be done by using (8), leading to following observation model

$$
H_{1}=\left[\begin{array}{lll}
\frac{\partial \epsilon}{\partial \hat{V}_{T A S}} & \frac{\partial \epsilon}{\partial \hat{W}_{N}} & \frac{\partial \epsilon}{\partial \hat{W}_{E}}
\end{array}\right],
$$

where $\epsilon$ is always estimated to be zero, i.e.

$$
\hat{X}_{1}^{+}=\hat{X}_{1}+K_{1}(0-\epsilon),
$$

where the superscript ${ }^{+}$indicates the a posteriori value of the state vector and $K_{1}$ is the corresponding Kalman gain

$$
K_{1}=P_{1} H_{1}^{T}\left(H_{1} P_{1} H_{1}+R_{1}\right)^{-1},
$$

where $P_{1}$ is the associated covariance matrix to the state vector $\hat{X}_{1}$ and $R_{1}$ corresponds to the uncertainty for the measurement of $\epsilon$. 


\section{B. Net force estimation}

The net force on the longitudinal axis of the aircraft can be estimated from the estimation of the true airspeed when the vehicle is close to level cruise flight. We consider the following state vector for the second Kalman filter in the Figure 5

$$
\hat{X}_{2}=\left[\begin{array}{ll}
\hat{V}_{T A S}^{-} & F_{N E T}
\end{array}\right]^{T}
$$

where $\hat{V}_{T A S}^{-}$is the true airspeed at the time instant $k-1$. Therefore we can calculate

$$
F_{N E T}(k)=m_{a c} \frac{\hat{V}_{T A S}(k)-\hat{V}_{T A S}^{-}(k)}{\Delta T},
$$

where $\Delta T=0.2$ seconds (corresponding to the frequency of the GPS employed in the first Kalman filter) and $m_{a c}$ is the mass of the aircraft. If we assume that the net force is kept constant between two consecutive time instants, then we can write the following dynamics for the second Kalman filter

$$
\begin{aligned}
X_{2}(k+1) & =\left[\begin{array}{cc}
0 & 0 \\
-\frac{m_{a c}}{\Delta T} & 0
\end{array}\right] X_{2}(k)+\left[\begin{array}{c}
1 \\
\frac{m_{a c}}{\Delta T}
\end{array}\right] u(k) \\
& =F_{2} X_{2}(k)+G_{2} u(k)
\end{aligned}
$$

where the input to the filter $u_{k}$ corresponds to the output $\hat{V}_{T A S}(k)$ from the first Kalman filter. Note that since the variance of $\hat{V}_{T A S}(k)$ is not constant, the dynamics of the covariance associated to $X_{2}$ is given by

$$
P_{2}(k+1)=F_{2} P_{2}(k) F_{2}^{T}+G_{2} \sigma_{\hat{V}_{T A S}}^{2}(k) G_{2}^{T},
$$

where the variance $\sigma_{\hat{V}_{T A S}}^{2}$ can be identified from the covariance matrix $P_{1}$. We have not included any correction stage in this second Kalman filter.

\section{Fusion of gaussian signals to find drag force}

The drag force can be estimated from the estimated net force and its variance calculated in the previous section and the direct measurements of the thrust force on the aircraft. Actually, the thrust force has been (indirectly) measured by two different sources as indicated in Figure 5. The first source comes from the characterization of the propeller in the wind tunnel, which needs as an input $\hat{V}_{T A S}$ from the first Kalman filter in Figure 5 . The second source is obtained from the (direct force) pressure sensor readings. It is located on top of the motor plate as depicted in Figure 6 and measures the sum of the propeller thrust $F_{\text {PropellerThrust }}$, and the force generated by the motor plate block under acceleration $F_{\text {motorplate }}=\vec{a} m_{\text {motorplate }}$. The estimated value of the thrust by this second source is just the subtraction of the two estimated values, $F_{\text {DirectSensor }}=$

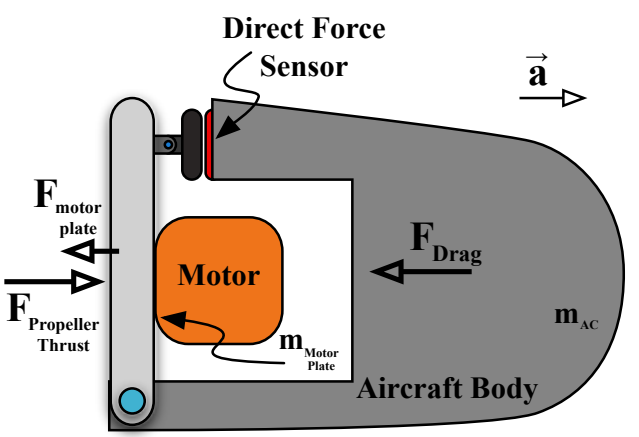

Figure 6. Net forces. $F_{\text {PropellerThrust }}-F_{\text {motorplate }}$, while its variance is the addition of the two variances associated to the force by the mounting plate and the force read by the sensor.

The two measured thrust forces are assumed to be Gaussian signals, therefore the fusion of these signals is given by the following expression

$$
F_{\text {thrust }}=\frac{f_{1} \sigma_{f_{2}}^{2}+f_{2} \sigma_{f_{1}}^{2}}{\sigma_{f_{1}}^{2}+\sigma_{f_{2}}^{2}}, \quad \sigma_{F_{\text {thrust }}}^{2}=\frac{\sigma_{f_{1}}^{2} \sigma_{f_{2}}^{2}}{\sigma_{f_{1}}^{2}+\sigma_{f_{2}}^{2}},
$$

where $f_{1}$ and $f_{2}$ are the thrust forces measured by the characterization of the propeller and the pressure sensor respectively, and $\sigma f_{1}^{2}+\sigma_{f_{2}}^{2}$ are the respective variances of $f_{1}$ and $f_{2}$.

Finally, the drag force and its uncertainty is estimated by the relation

$$
F_{\text {drag }}=F_{N E T}-F_{\text {thrust }}, \quad \sigma_{F_{\text {drag }}}^{2}=\sigma_{F_{N E T}}^{2}+\sigma_{F_{\text {thrust }}}^{2},
$$

note that since the variances of the states in the Kalman filter are time-varying, the uncertainty of $F_{d r a g}$ is also time-varying as we will see in the following section of experimental results. 


\section{Experimental Results}

Several flight tests and ground calibration tests have been conducted. All of these data and plotting scripts have been made available for public in https://github.com/mrtbrnz/FlightEx.git. A typical flight test profile is shown in Figure 7. First graph shows the ground speed measurements obtained by the onboard GPS sensor and the estimated airspeed from the filter presented in the previous section. The second graph shows the GPS altitude in meters above the sea level, and the third graph shows the motor RPM measured from the on-board speed controller. It can be seen that both altitude, airspeed, and the motor RPM are kept constant during the whole flight tests except from take-off and landing phases. It should also be noted that the complete flight was done in fully autonomous mode.
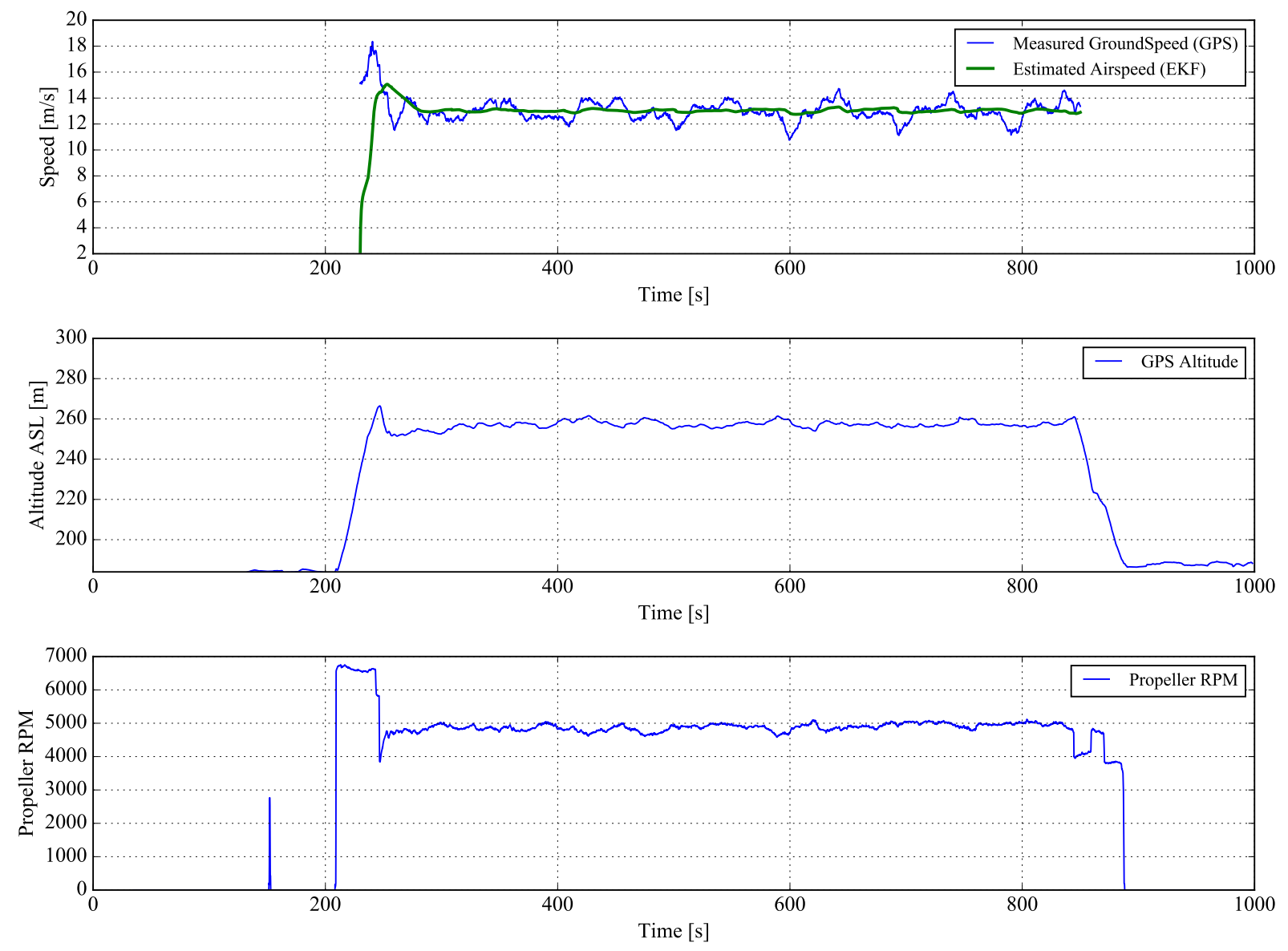

Figure 7. Basic flight informations of a typical flight experiment, after the take-off the altitude during the whole test remains almost constant, likewise for the propeller rotation speed and estimated airspeed.

The measured output from the direct force sensor is shown in the Figure 8. The measurement noise is very significant, and at the first sight one can think it is a meaningless signal. The main sources of vibration could be coming from the sensor itself, analog-to-digital converters, external vibrations of the vehicle and also the effect that the wake of the fuselage is being cut by the propeller twice at each rotation. Additionally, the direct force measurement mechanism has a bottom hinge with bearings, so every force is generating a torque and then captured by the pressure sensor at the high end of the mechanism.

In order to avoid vibrations several methods are tested. The pressure sensor initinally was being pressed between two smooth metallic surfaces, but this was later modified by adding a $1 \mathrm{~mm}$ thick double-sided tape with the objective of absorbing some of the vibrations. This had almost no effect on the signal noise. The motor mount was rigidly connected to the motor in a first design, but later modifed by adding rubber vibration isolators. However this last addition increased the vibrations even more. Pre-loading the system with an elastic band or a metal spring resulted the best among all the designs.

Further investigation of the vibration problem showed that the main source was coming from the propeller 


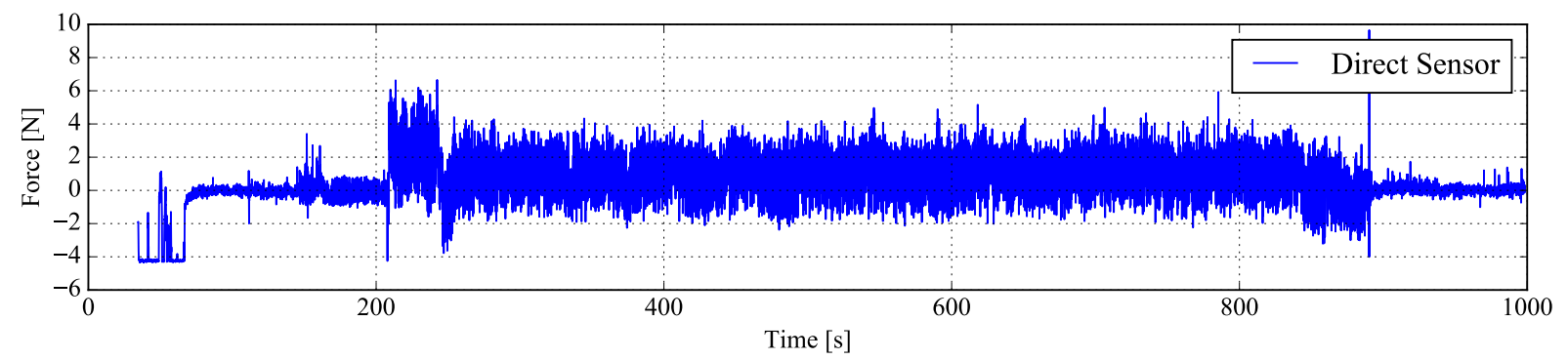

Figure 8. Showing the calibrated direct force sensor measurement outputs.

and fuselage wake interaction. Each time when the propeller blade travels along the fuselage-wing wake, it experiences a pressure jump because of the shear layer, and this results a torque hence a force on the direct force sensor. This effect can be clearly seen in the Figure 9. The aircraft is first powered on the ground in static condition while being held by two people from the wings; and by keeping constant the throttle command, the aircraft is placed into the wind-tunnel test section. The increasing of the vibration level, the propeller RPM and the reduction on the measured force are clearly visible. This basically proves that the noisy signal is actually carrying useful information of the force measurements that should not be omitted.
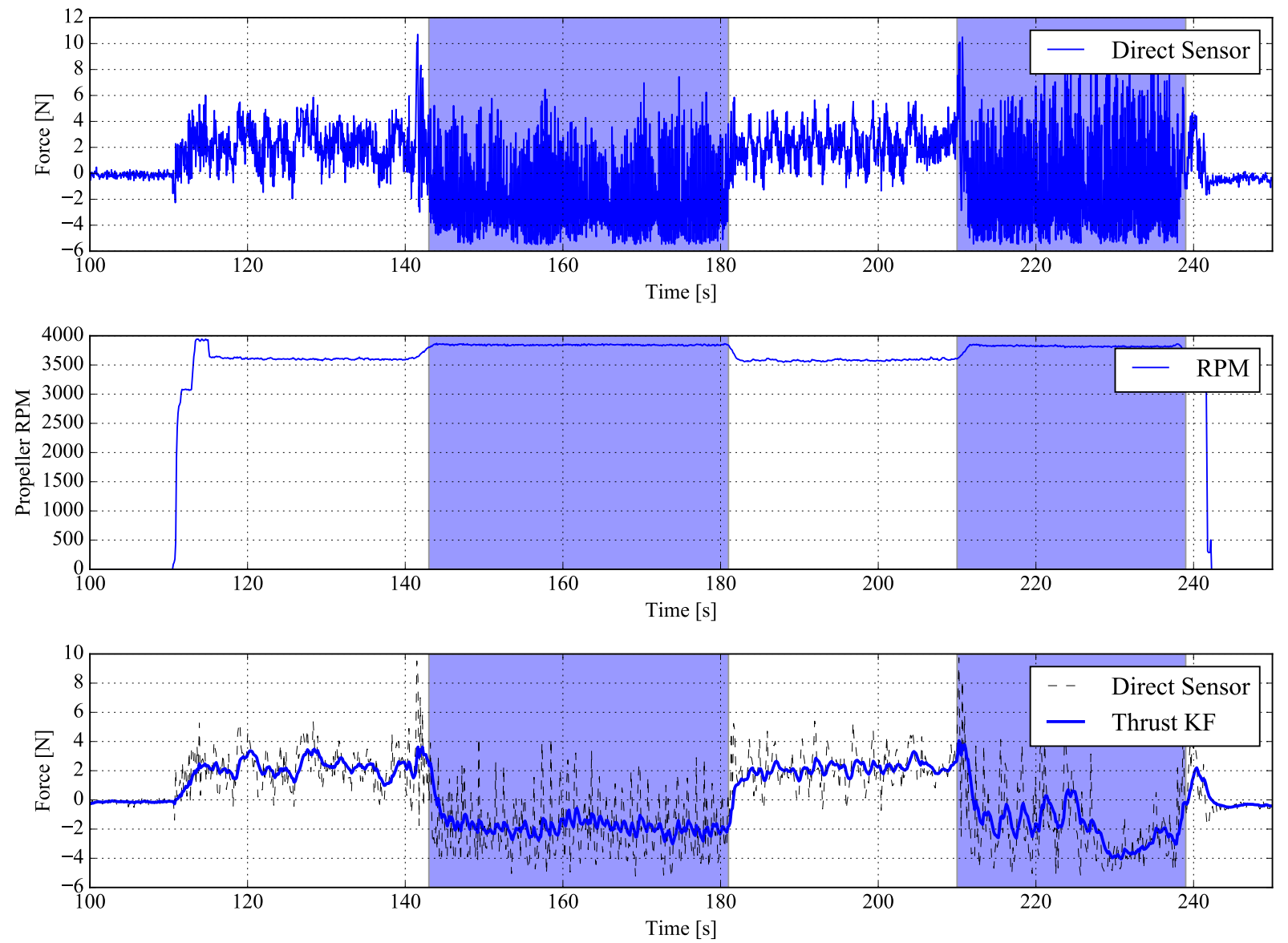

Figure 9. Investigation of fuselage wake and propeller interaction is shown, the blue shaded sections are showing when the aircraft is inside the wind-tunnel, the existing of significant interaction is clearly visible with increased amplitude of the signal. 


\section{A. In-Flight Thrust and Drag Force Measurements}

Figure 10 shows the estimated forces during a short period of the flight shown in Figure 7. The dashed black line shows the direct force measurements, the red line shows the resultant in-flight thrust estimation and the green line shows the estimated drag force. Additionally, the propeller thrust force estimation is plotted in blue based on the wind tunnel characterized function that takes the estimated airspeed and the measured RPM as inputs. As mentioned before, high frequency vibrations show the interaction between the fuselage wake and the propeller. The average thrust and drag force is approximately $1 N$ and $-1 N$ respectively.

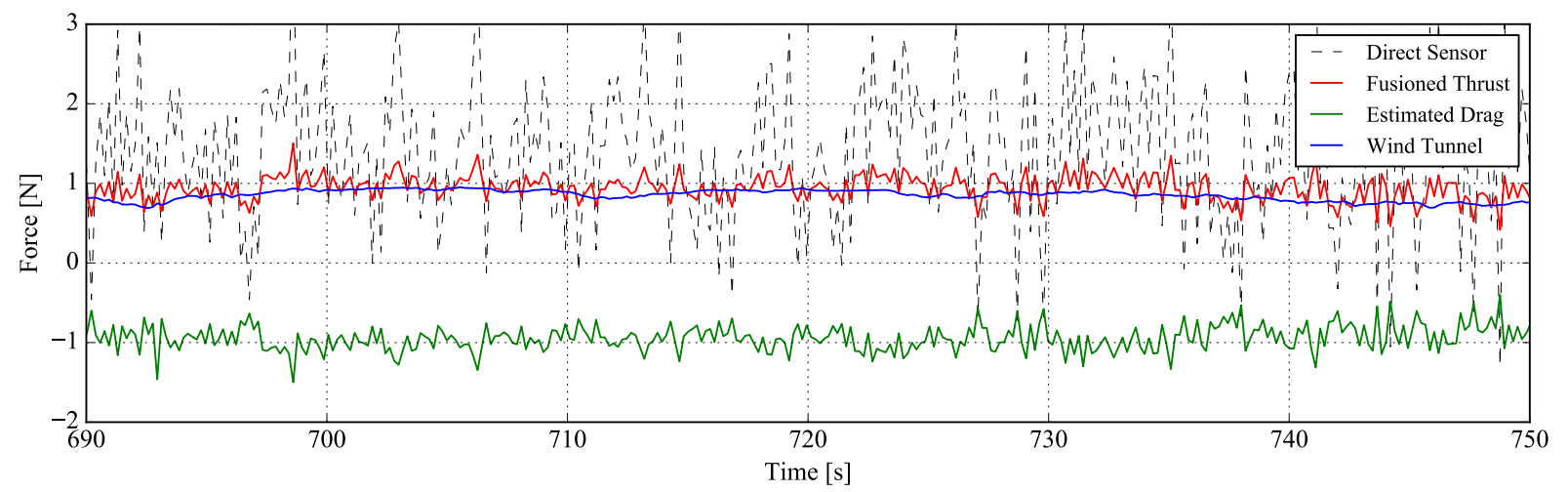

Figure 10. Result of information fusion for thrust and drag force estimation.

Once the drag force is estimated, additional variables can be investigated, such as the aerodynamic power generated by the propeller $P_{\text {Aero }}=\hat{V}_{T A S}$ Thrust. The propulsion system efficiency can also be extracted in real-time, with the ratio of aerodynamic power over electrical power $\eta=P_{\text {Aero }} / P_{\text {Electrical }}$. These variables are plotted in Figure 11. The total efficiency of the propulsion system varies between $30-50 \%$ which is wide range however averaging around $40+\%$ falls into the correct value for this propulsion couple according to previous experiences.

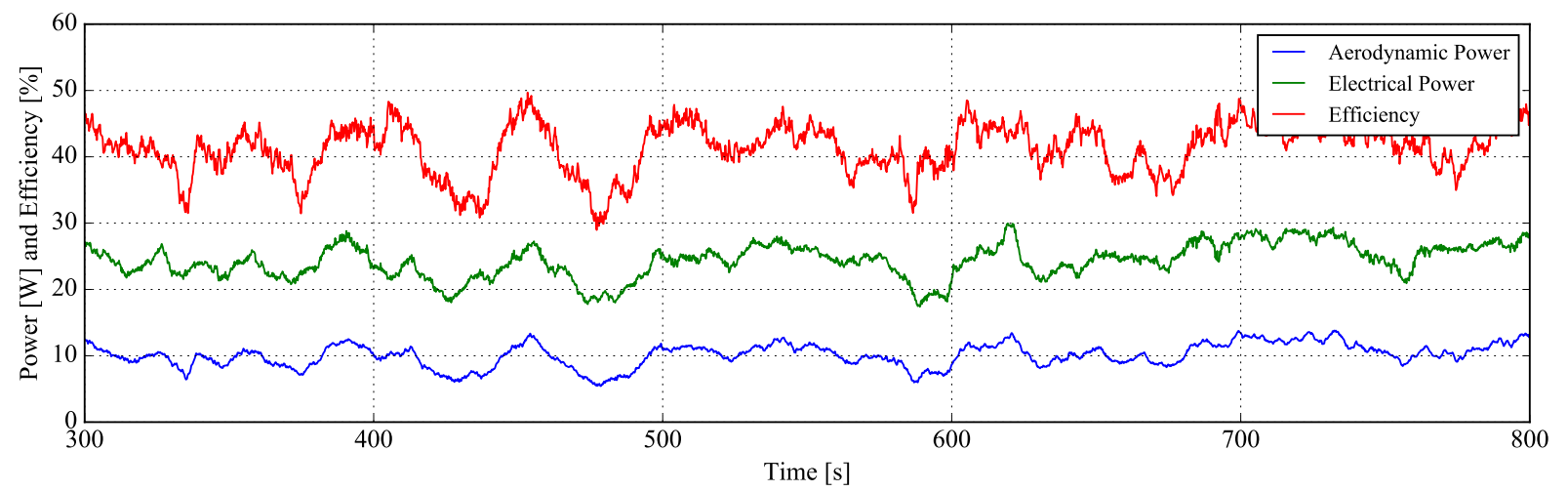

Figure 11. Aerodynamic power, electrical power and the instantaneous propulsion system efficiency are shown.

According to the estimated flight speed of $\hat{V}_{T A S}=13.1$ during the cruise flight phase, the resultant lift coefficient will be around $C_{L}=0.35$. The corresponding drag coefficient is around $C_{D}=0.029$ as shown on the histogram in Figure 12. The aircraft used in the flight tests has already been characterized at different flight speeds by using glide tests as described in. ${ }^{9}$ However during the glide phase, the propeller was free-wheeling and generating some additional drag. This is clearly visible in Figure 4, where the propeller generates nearly $0.6 \mathrm{~N}$ of negative thrust when it is not powered. If this additional drag is removed, as a correction, from the estimated drag coefficient in, ${ }^{9}$ the resultant drag coefficient becomes approximately $C_{D}=0.03$ for the given lift coefficient of $C_{L}=0.35$. This gives us confidence for the two characterization methods. 


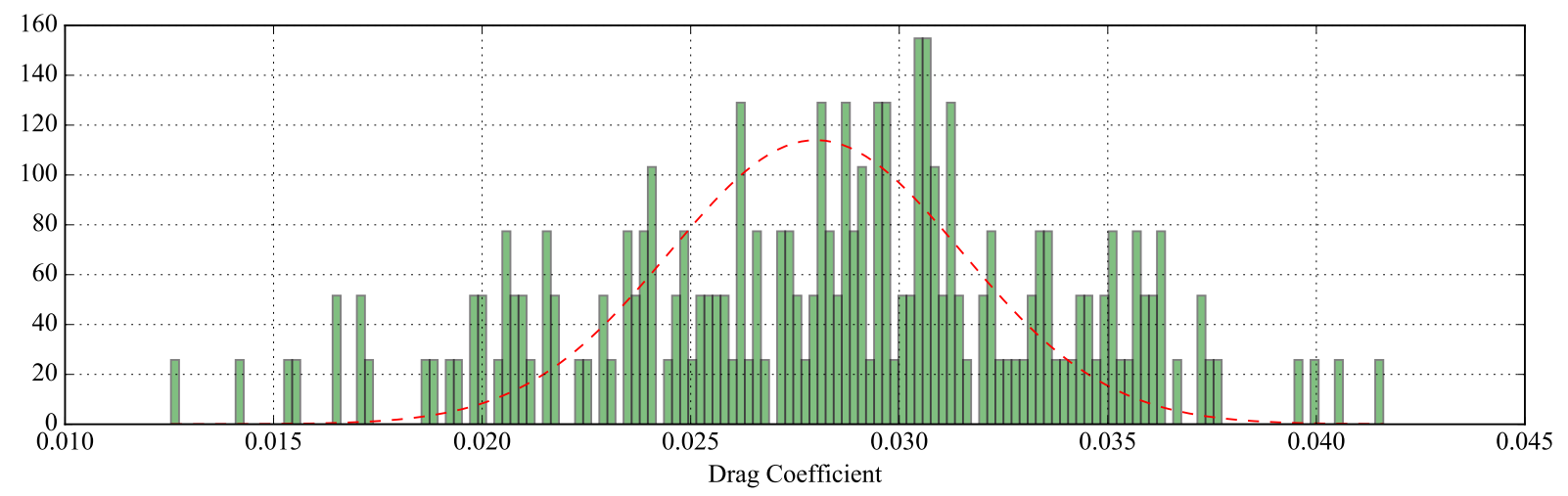

Figure 12. Drag Coefficient histogram over a short period taken from the real flight test.

\section{Conclusion and Future Work}

An alternative estimation method of in-flight thrust and drag force has been presented. Two sources of indirect thrust measurements are fused for a better in-flight thrust and drag force estimation. The propulsion system has been characterized in the wind-tunnel in order to obtain an analytic function for the thrust force. Measured propeller RPM and estimated airspeed have been used as inputs for this function in order to obtain the first source of thrust estimation. The second source comes from an on-board sensor that measures the sum of propeller thrust force and the reaction force of the motor plate generated because of an accelerating frame. The resultant thrust and drag forces from this fusion showed additional high frequency features generated by the interaction of fuselage wake and propeller. The fluctuations of forces during the flight makes it difficult to obtain a fixed value, however averaged values are usable for the thrust force, drag force, and the drag coefficient. These can be used for modeling and performance calculations of propeller driven small UAVs.

\section{Acknowledgments}

The authors would like to thank to Antoine Drouin for the explanations and discussion on Kalman filter, Michel Gorraz for building the electric circuit, Alexandre Bustico for debugging the ESC telemetry module code, and Xavier Paris for his support during the whole flight tests.

\section{Appendix}

The thin film force sensor reduces its resistance when a pressure is applied on it. However, the calibration curve using the resistance is cumbersome, therefore as suggested by the supplier, a dedicated electronics board has been build in order to measure conductance instead of resistance, which has a linear relation, making it easier to calibrate. Figure 13 shows the schematics of the board. In the presented application, the resistance values are respectively $R_{1}=1 K \Omega, R_{2}=4.7 \mathrm{~K} \Omega$, and $R_{F}=47 \mathrm{~K} \Omega$.

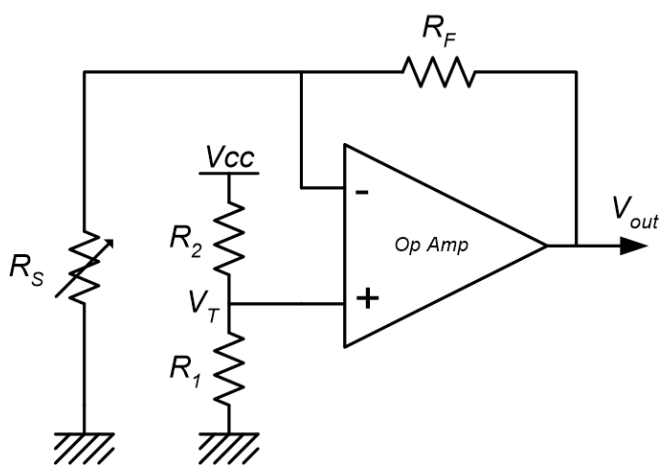

Figure 13. Schematics of the dedicated electronic circuit. 


\section{References}

${ }^{1}$ Roberts, G., Calmer, R., et al., "multi-dimensional Cloud-aERosol Exploratory Study using RPAS (mCERES): Bottomup and top-down closure of aerosol-cloud interactions," EGU General Assembly Conference Abstracts, Vol. 18, 2016, p. 6530.

${ }^{2}$ Bevacqua, G., Cacace, J., Finzi, A., and Lippiello, V., "Mixed-Initiative Planning and Execution for Multiple Drones in Search and Rescue Missions." ICAPS, 2015, pp. 315-323.

3 "SHERPA Project," june 2016.

${ }^{4}$ Bronz, M. and Hattenberger, G., "Design of A High-Performance Tailless MAV Through Planform Optimization," 33rd AIAA Applied Aerodynamics Conference, AIAA, 2015, pp. eISBN-978.

${ }^{5}$ Nio, J., Mitrache, F., Cosyn, P., and Keyser, R. D., "Model Identification of a Micro Air Vehicle," Journal of Bionic Engineering, Vol. 4, No. 4, 2007, pp. $227-236$.

${ }^{6}$ Valavanis, K. P. and Vachtsevanos, G. J., Handbook of Unmanned Aerial Vehicles, chap. UAV Modeling, Simulation, Estimation, and Identification: Introduction, Springer Netherlands, Dordrecht, 2015, pp. 1215-1216.

${ }^{7}$ Simsek, O. and Tekinalp, O., "System Identification and Handling Quality Analysis of a UAV from Flight Test Data," AIAA Atmospheric Flight Mechanics Conference, AIAA SciTech, AIAA 2015, 2015.

${ }^{8}$ Edwards, D., "Performance Testing of RNR's SBXC Using a Piccolo Autopilotd," Tech. rep., 2007.

${ }^{9}$ Bronz, M. and Hattenberger, G., "Aerodynamic Characterization of an Off-the-Shelf Aircraft via Flight Test and Numerical Simulation," AIAA Flight Testing / Ground Testing Conference, Washington, DC, AIAA, June 2016.

${ }^{10}$ Stoll, A. M., Bevirt, J., Moore, M. D., Fredericks, W. J., and Borer, N. K., "Drag Reduction Through Distributed Electric Propulsion," Aviation Technology, Integration, and Operations Conference, Atlanta, Georgia, 16-20 June 2014.

${ }^{11}$ Brisset, P., Drouin, A., Gorraz, M., Huard, P.-S., and Tyler, J., "The paparazzi solution," MAV2006, Sandestin, Florida, 2006.

${ }^{12}$ Hattenberger, G., Bronz, M., and Gorraz, M., "Using the Paparazzi UAV System for Scientific Research," IMAV 2014, International Micro Air Vehicle Conference and Competition 2014, Delft, Netherlands, Aug. 2014, pp. pp 247-252.

${ }^{13}$ Hattenberger, G., Drouin, A., and Bronz, M., "Electric Propulsion System Characterization through Experiments," IMAV 2016, International Micro Air Vehicle Conference and Competition, Beijing, China, 2016.

${ }^{14}$ Edwards, D. J., "Autonomous Locator of Thermals (ALOFT) Autonomous Soaring Algorithm," Tech. rep., DTIC Document, 2015.

${ }^{15}$ Kahn, A. D. and Edwards, D. J., "Navigation, guidance and control for the CICADA expendable micro air vehicle," AIAA Guidance, Navigation, and Control Conf., 2012. 